\title{
REAÇÕES DE CONTRAÇÃO DE ANEL PROMOVIDAS POR SAIS DE TÁLIO(III)
}

Helena M. C. Ferraz* e Luiz F. Silva Jr.

Instituto de Química - Universidade de São Paulo - CP 26077 - 05599-970 - São Paulo - SP

Recebido em 16/3/99; aceito em 9/6/99

\begin{abstract}
RING CONTRACTION REACTIONS PROMOTED BY THALLIUM(III) SALTS. Thallium(III) salts promote a number of different reactions useful in organic synthesis. In this paper, the ring contraction of ketones and olefins, mediated by thallium(III) salts, is exhaustively reviewed.
\end{abstract}

Keywords: thallium(III) salts; ring contraction; oxidative rearrangement.

\section{INTRODUÇÃO}

O número de publicações relacionadas com o uso de tálio(III) para promover transformações em moléculas orgânicas teve seu ápice na década de 70 , quando foram realizadas inúmeras reações com uma grande variedade de moléculas orgânicas. Nos últimos anos, o número de trabalhos voltou a crescer, após uma queda ao longo da década de $80^{1}$.

Existem inúmeros artigos de revisão abordando o uso de tálio(III) em síntese orgânica ${ }^{1-19}$. Um dos artigos mais importantes, e dos mais citados, foi publicado por McKillop e Taylor ${ }^{8} \mathrm{em}$ 1982. Esta revisão, com 231 referências, mostra com clareza o uso de compostos de tálio em síntese orgânica. Posteriormente, três artigos foram publicados por nós ${ }^{12,15,16} \mathrm{em}$ Química Nova.

Apesar do grande número de revisões sobre tálio(III) em síntese orgânica, nenhuma delas aborda especificamente as reações de contração de anel. Além disso, o número de artigos sobre esta reação, publicados desde as últimas revisões, corresponde a quase $40 \%$ do número total.

A presente revisão engloba os trabalhos publicados do início da década de 60 até o ano de 1998, e está dividida em dois itens: o primeiro aborda a reação de contração de anéis de cetonas (incluindo as $\alpha, \beta$-insaturadas), enquanto que o segundo trata da contração de anéis contendo ligações duplas.

Durante o texto, os sais de tálio(III) mais utilizados serão chamados pelas seguintes abreviaturas: triacetato de tálio, TTA; trinitrato de tálio, TTN; e trifluoracetato de tálio, TTFA.

\section{CONTRAÇÃO DE ANÉIS CONTENDO CARBONILA}

\section{Cicloexanonas}

O primeiro trabalho que descreveu a contração de uma cetona cíclica, usando tálio(III) como oxidante, foi publicado em 1966 por Wiberg e $\operatorname{Koch}^{20}$. Trata-se da reação da cicloexanona com $\mathrm{Tl}(\mathrm{III})$, em solução de ácido perclórico 35\%, fornecendo como produto principal o ácido ciclopentanocarboxílico em $75 \%$ de rendimento (Equação 1).

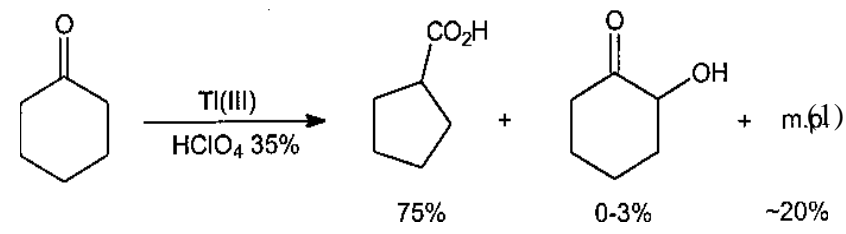

A contração da cicloexanona ocorre, segundo os autores, por um mecanismo onde o primeiro passo seria a formação de um derivado $\alpha$-taliado, que após ataque da água à carbonila, sofreria um rearranjo, fornecendo o ácido ciclopentanocarboxílico (Esquema 1).

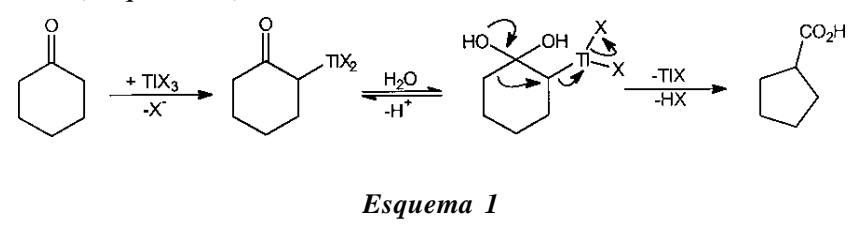

A reação de alquilcicloexanonas com tálio(III) também foi descrita nessa comunicação (Tabela 1). Cabe ressaltar que a 2,2dimetilcicloexanona levou ao ácido 2,2-dimetilciclopentanocarboxílico, enquanto que a 2-metilcicloexanona não forneceu o produto de contração. Infelizmente, não foram fornecidos diversos dados nesse trabalho, tais como rendimentos, tempos de reação e configuração relativa dos produtos formados. A contração de ciclopentanona, cicloeptanona e ciclooctanona aos respectivos ácidos não ocorreu nas condições utilizadas.

Em 1972, McKillop et al. ${ }^{21}$ também estudaram a reação da cicloexanona com tálio(III), porém utilizando TTN em ácido acético glacial. O ácido ciclopentanocarboxílico foi obtido em $84 \%$ de rendimento (Equação 2).<smiles>O=C1CCCCC1</smiles>

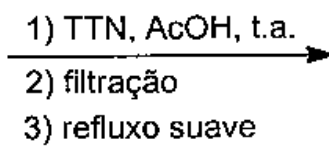

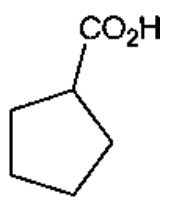

$84 \%$
Os autores propuseram, nesse artigo, um mecanismo diferente daquele de Wiberg (cf. Esquema 1). A principal diferença consiste na formação de 1 como intermediário (Esquema 2).

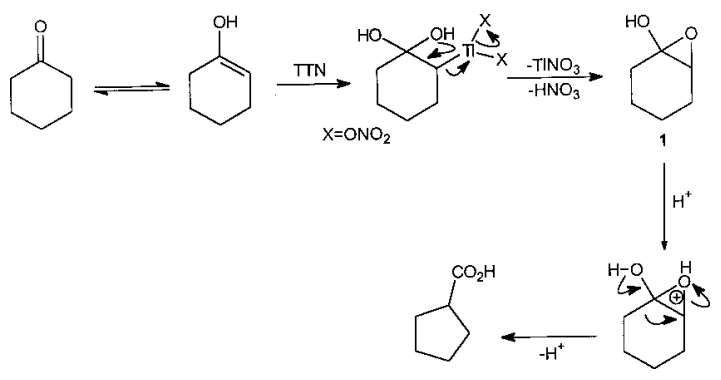

e-mail: hmferraz@quim.iq.usp.br 
Tabela 1. Reação de Alquilcicloexanonas com Tálio(III) em $\mathrm{HClO}_{4} 35 \%$.

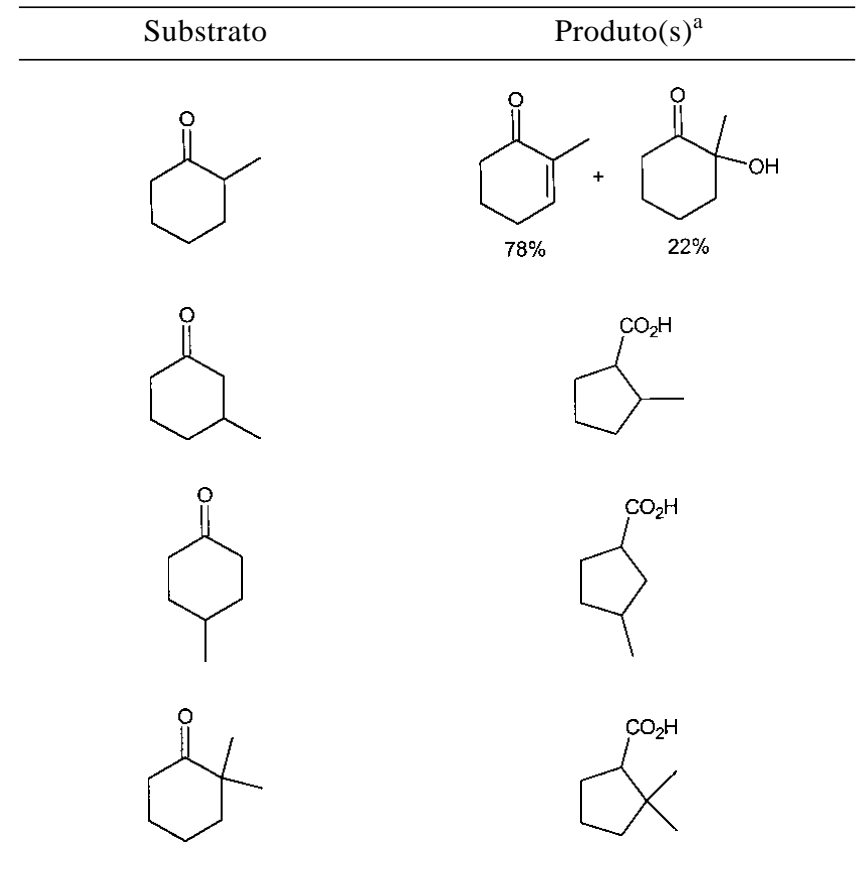

a rendimentos não fornecidos.

Cabe lembrar que a reação de cicloexanonas com tálio(III) levou a outros produtos, quando diferentes condições experimentais foram utilizadas ${ }^{21-25}$. Khanna et al. ${ }^{24}$, por exemplo, obtiveram $\alpha$-mesiloxi-cetonas, ao realizarem a reação da 2metilcicloexanona com TTA e ácido metanossulfônico em acetonitrila (Equação 3).<smiles>COC1CCCC(C)C1=O</smiles>

A reação de uma série de enaminas cíclicas com TTA levou a $\alpha$-acetoxi-cetonas em rendimentos de baixos a bons ${ }^{26,27}$. Esta reação foi realizada em diferentes solventes, como clorofórmio, ácido acético e benzeno. Exemplos representativos são mostrados no Esquema 3.<smiles>[R]C1=CCCCC1</smiles><smiles>[R]C1=CCC(C(C)(C)C)CC1</smiles>

Esquema 3

A contração da cicloexanona 2 levou ao éster 3, com rendimento moderado ${ }^{28}$ (Equação 4). Além disso, outros produtos de rearranjo foram observados em menor proporção (3 a 8\%).
Recentemente, a reação de alquilcicloexanonas com TTN foi reinvestigada ${ }^{29}$ (Tabela 2).

Tabela 2. Reação de Alquilcicloexanonas com TTN. $3 \mathrm{H}_{2} \mathrm{O}$ em $\mathrm{CH}_{2} \mathrm{Cl}_{2}$ a $25^{\circ} \mathrm{C}$.<smiles>CC1CCC(=O)CC1</smiles><smiles>CC(C)(C)C1CCCC(=O)C1</smiles>

b<smiles>CC(C)(C)C1CCC(C(=O)O)C1</smiles><smiles>CC1CCCCC1=O</smiles>

$\mathrm{c}$

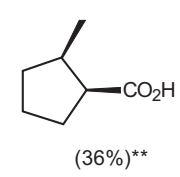

*Condições: a) 1,1 eq. TTN 1 dia; b) 1,5 eq. TTN 2 dias; c) 2 eq. TTN 3 dias; ${ }^{* *}$ determinado por C. G.

A diastereosseletividade observada nestas reações é melhor explicada pelo mecanismo de $\mathrm{McKillop}^{21}$ (Esquema 2) do que pelo de Wiberg ${ }^{20}$ (Esquema 1), já que a contração da 4-metilcicloexanona, por exemplo, levaria ao ácido cis segundo o mecanismo de McKillop, e ao ácido trans segundo Wiberg (Esquema 4).

\section{Cetonas Bicíclicas}

Recentemente, Bird e Cooper ${ }^{30,31}$ obtiveram o sistema cishidrindânico pela contração da decalona 4, utilizando TTN em ácido acético glacial (Equação 5).

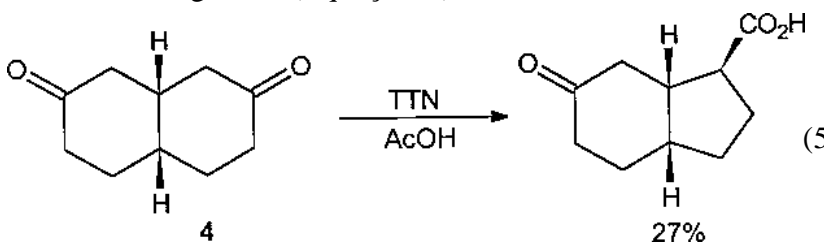

Já a reação de trans-2-decalonas com TTN, utilizando diclorometano como solvente, forneceu os correspondentes 


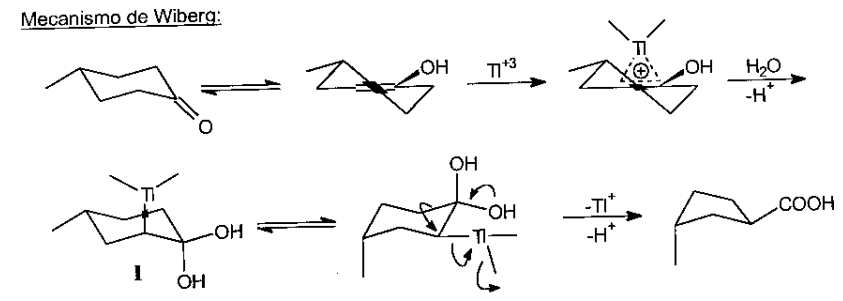

Mecanismo de McKillop:

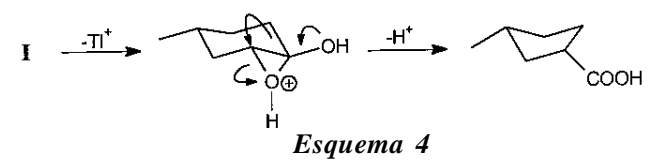

produtos de contração com excelentes rendimentos e diastereosseletividade, desde que não houvesse um grupo metila em $\alpha$ à carbonila ${ }^{32}$ (Tabela 3 ).

Octalonas forneceram produtos de contração quando tratadas com TTN em uma mistura de trimetilortoformiato (TMOF) e metanol ${ }^{33}$, mas quando se utilizou TTA, foram obtidos produtos de desidrogenação ${ }^{34}$ (Esquema 5).<smiles>O=C1CCCC2=C1CCCC2</smiles>

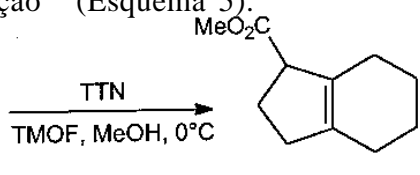<smiles>CC12CCCCC1=CC(=O)CC2</smiles><smiles>CO[C@H](C(C)O)[C@H]1C=C2CCCCC2(C)C1</smiles><smiles>CC1=C2CCCCC2(C)CCC1=O</smiles>

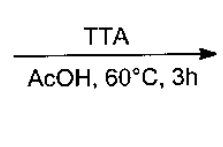<smiles>CC1=C2CCCCC2(C)C=CC1=O</smiles>

Esquema 5

Utilizando TTN adsorvido em K-10 (Montmorilonite), Taylor et al. ${ }^{35}$ realizaram a contração da 1-tetralona, obtendo o 1-indanocarboxilato de metila, juntamente com a 2-metoxi-1tetralona. A reação deste mesmo substrato com TTN em metanol levou a uma mistura de mais de dez produtos ${ }^{35}$, enquanto que a reação da 6-metoxi-1-tetralona com TTN em ácido acético forneceu a 2,2-dinitrato-6-metoxi-1-tetralona em baixo rendimento ${ }^{36}$ (Esquema 6).<smiles>O=C1CCCc2ccccc21</smiles><smiles>CC(C)ON1OCCO1</smiles><smiles>CC(=O)C1CCc2ccccc21</smiles><smiles>COC1CCc2ccccc2C1=O</smiles><smiles>COc1ccc2c(c1)CCCC2=O</smiles><smiles>COc1ccc2c(c1)CCC(O)(O[N+](=O)[O-])C2=O</smiles>

Esquema 6

Uma característica do TTN/K-10 é que, nas condições de reação, tanto o TTN quanto o $\mathrm{TlNO}_{3}$ que se forma estão firmemente ligados ao suporte e, consequentemente, não há contaminação (medida por absorção atômica) do solvente nem do produto ${ }^{35}$.
Tabela 3. Reação de trans-2-decalonas com TTN. $3 \mathrm{H}_{2} \mathrm{O}$ em $\mathrm{CH}_{2} \mathrm{Cl}_{2}$ a $25^{\circ} \mathrm{C}$<smiles>CC[C@]1(C(C)C)CC(=O)CC[C@]1(C)C1CC(=O)CCC1(C)C</smiles><smiles>C[C@H]1CC(=O)C[C@H]2CCCC[C@@]12C</smiles>
mistura complexa

A reação de uma série de cromanonas foi estudada em 1982 por Ciattini et al. ${ }^{37}$. Quando a reação foi realizada com TTN em metanol acidificado com ácido perclórico, foi observada a formação de produtos de desidrogenação, enquanto que a reação com TTN em TMOF forneceu produtos de contração e de $\alpha$-metoxilação (Esquema 7).

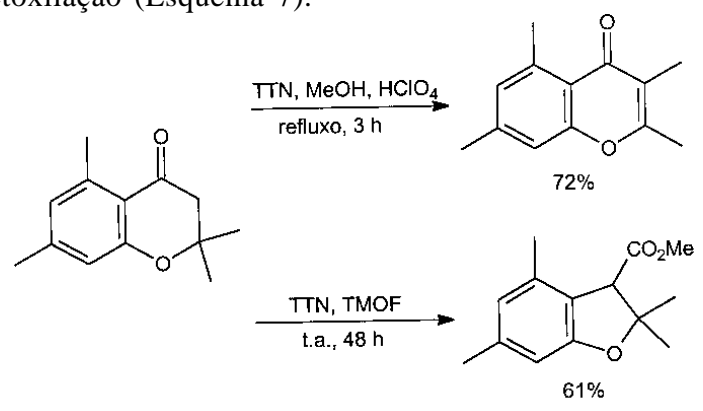

Esquema 7 
Analogamente ao observado com as cromanonas, o rearranjo oxidativo de flavanonas mediado por TTN, na presença de $\mathrm{HClO}_{4} / \mathrm{TMOF}$, forneceu 2,3-desidro-2-arilbenzofuran-3-carboxilatos de metila como principais produtos ${ }^{38}$ (Equação 6). A configuração relativa dos produtos não foi fornecida.<smiles>CC(=O)C1c2ccccc2OC1C(=O)ON1C(=O)c2ccccc21</smiles>

Grisar et al. ${ }^{39}$ realizaram a contração das cetonas aromáticas 5 aos ésteres 6, utilizando TTN em TMOF/MeOH, como etapa na síntese de análogos do tocoferol (Esquema 8).<smiles>[R]Oc1c(C)c(C)c2c(c1C)C(=O)CC(C)(C)O2</smiles><smiles>CC(=O)C1c2c(C)c(O)c(C)c(C)c2OC1(C)C</smiles>

Esquema 8

Utilizando TTN em metanol como oxidante, Irwin e Jones ${ }^{40}$ mostraram que a reação de contração da (1S,5S)-biciclo[3.2.1]-2octanona pode ser realizada com bons rendimentos (Equação 7).

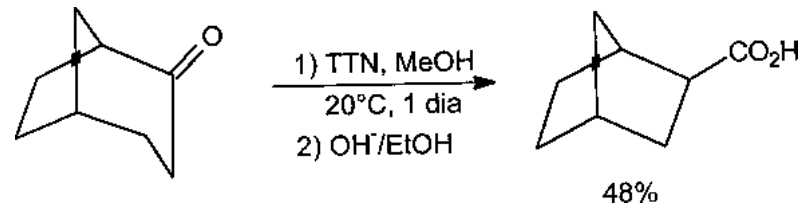

Just e Donnini ${ }^{41}$, entretanto, não tiveram o mesmo sucesso na contração de um sistema análogo a este, ao utilizarem TTN em ácido acético glacial (Equação 8).

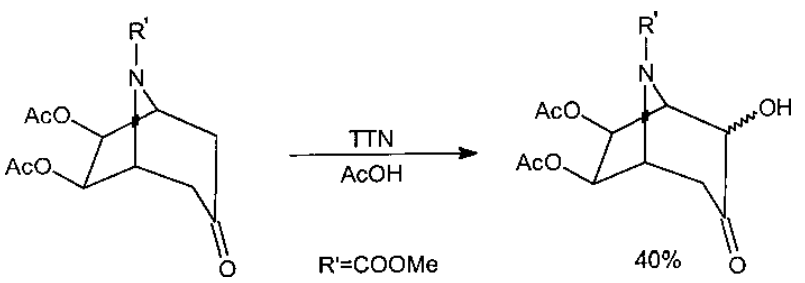

\section{Cetonas Esteroidais}

As reações de cetonas esteroidais com sais de tálio(III) fornecem produtos de contração, $\alpha$-oxidação e desidrogenação, conforme o substrato e as condições utilizadas.

Romeo e Ortar obtiveram o produto de contração ao reagirem o esteróide 7 com TTA em ácido acético ${ }^{42}$, enquanto que produtos de contração e desidrogenação foram formados para esteróides como 8 e $9^{43}$. No caso de $\Delta^{1}$ ou $\Delta^{4}$-3-ceto esteróides, como 10, por exemplo, foram obtidos apenas produtos de desidrogenação ${ }^{42}$ (Esquema 9).

Outro grupo de pesquisadores, entretanto, realizou a contração de $\Delta^{1}$-3-ceto esteróides, utilizando TTN em TMOF/ $\mathrm{MeOH}^{44}$ (Equação 9), de modo análogo à reação de octalonas (Esquema 5).

1,2-Dicetonas também levaram a produtos de contração, conforme relatado por Maione et al. ${ }^{45}$ (Equação 10).

Em um trabalho publicado em 1988, Auclair e Gramain ${ }^{46}$ realizaram a contração de um esteróide não natural, utilizando TTA como oxidante. Para explicar a estereoquímica do produto formado, os autores propuseram o intermediário 11 (Esquema 10).

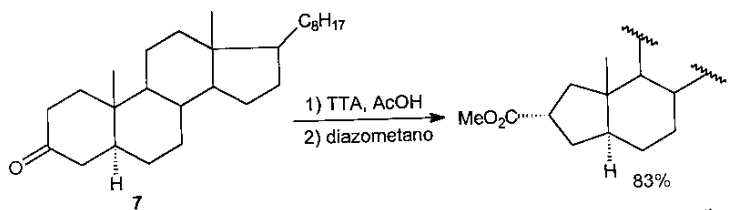

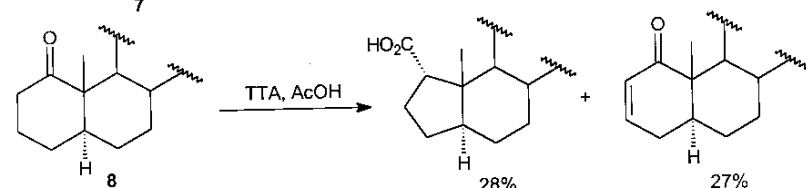

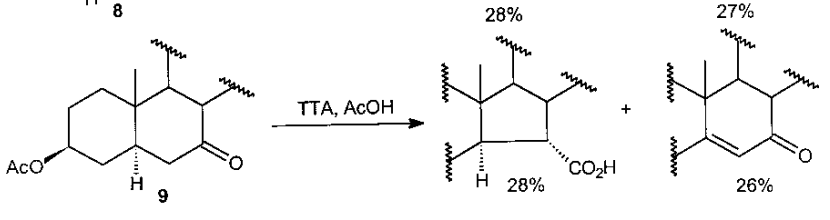

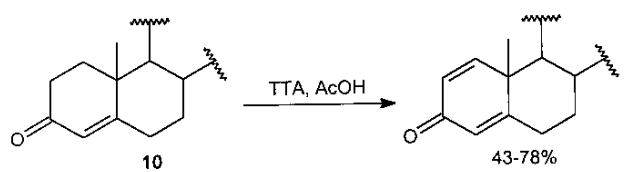

Esquema 9

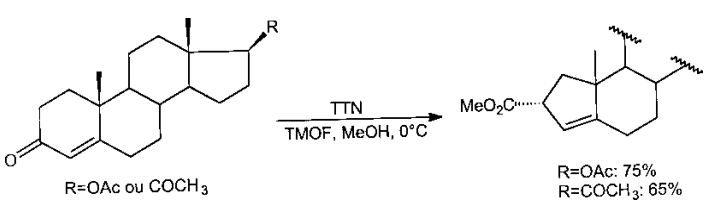

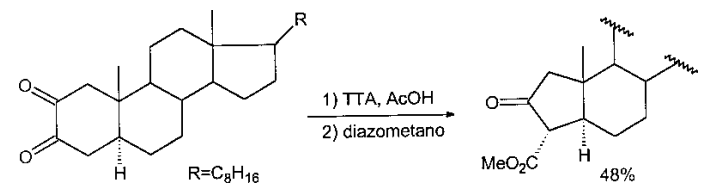

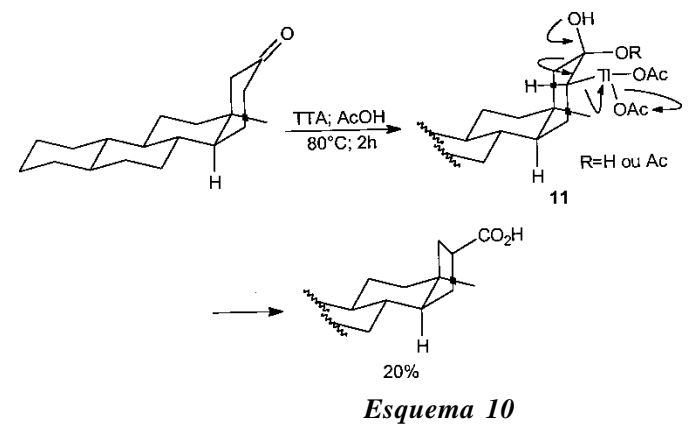

\section{Ciclobutanona}

A formação do ácido ciclopropanocarboxílico, através da contração da ciclobutanona com tálio(III), foi realizada com sucesso em duas condições diferentes ${ }^{47}$. Os autores aplicaram tanto a condição de Wiberg ${ }^{20}$ quanto a de McKillop ${ }^{21}$ (Esquema 11).

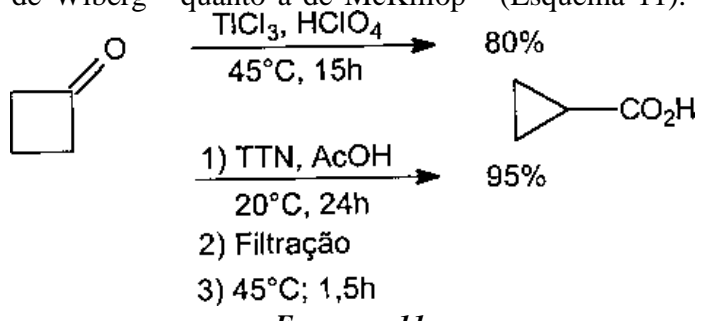




\section{CONTRAÇÃO DE ANÉIS CONTENDO LIGAÇÕES DUPLAS}

\section{Cicloexenos}

No início da década de 60 foram publicados diversos trabalhos sobre a reação do cicloexeno com sais de tálio(III) ${ }^{22,48-50}$.

O exemplo pioneiro na reação de contração de olefinas promovida por sais de tálio(III) foi realizado por Kabbe, que em 1962 relatou a oxidação do cicloexeno com TTA em dois solventes diferentes ${ }^{22}$. O principal produto obtido na reação em metanol foi o (dimetoximetil)-ciclopentano, enquanto que a reação em ácido acético levou a uma mistura do 1,2-diacetoxicicloexano e do (diacetoximetil)-ciclopentano (Esquema 12).

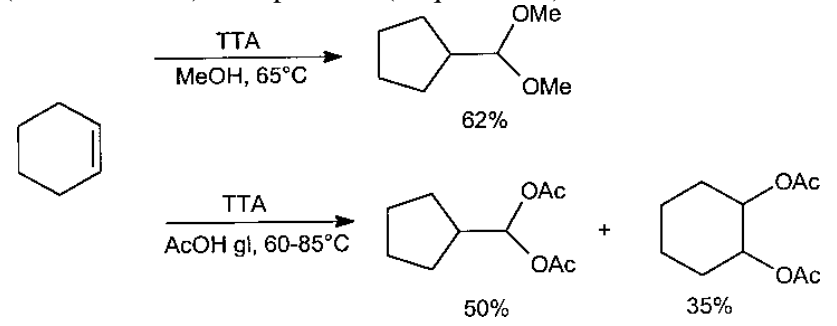

Esquema 12

Este trabalho, que trata também da oxidação de outros substratos, tornou-se um importante ponto de referência, sendo citado em inúmeros artigos.

No ano seguinte, Anderson e Winstein ${ }^{48}$ estudaram a reação do cicloexeno com TTA em ácido acético, obtendo como produtos o cis e trans-1,2-diacetoxicicloexano, o ciclopentanocarboxaldeído e o (diacetoximetil)-ciclopentano, além de 2-3-\% do $\mathrm{d}^{2}$-cicloexenilacetato (Equação 11). Estes resultados são similares aos relatados por Kabbe.

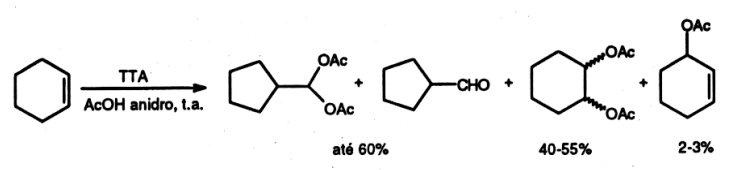

Lee e Price ${ }^{49,50}$ realizaram a reação do cicloexeno em condições análogas às anteriores, mas analisaram também o possível efeito da temperatura na distribuição dos produtos. $\mathrm{O}$ aumento da temperatura provocou um aumento na proporção do produto de oxidação alílica, e uma diminuição do produto de adição, porém não alterou a distribuição dos produtos de contração (Esquema 13).

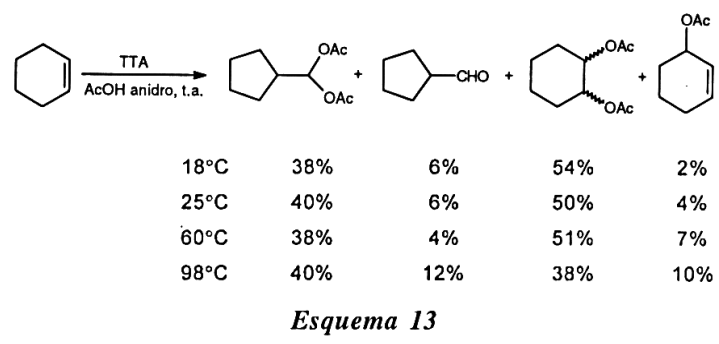

O início da década de 70 marca o surgimento de novos trabalhos sobre a contração de cicloexenos com sais de tálio(III).

A reação do cicloexeno com sulfato de tálio(III) em meio aquoso levou ao ciclopentanocarboxaldeído como produto principal, enquanto que a reação com 3 e 4-t-butilcicloexeno forneceu dióis trans como produtos majoritários, não sendo detectada a presença de produtos de contração ${ }^{51,52}$ (Esquema 14).

A reação do cicloexeno com sais de tálio pode ser direcionada

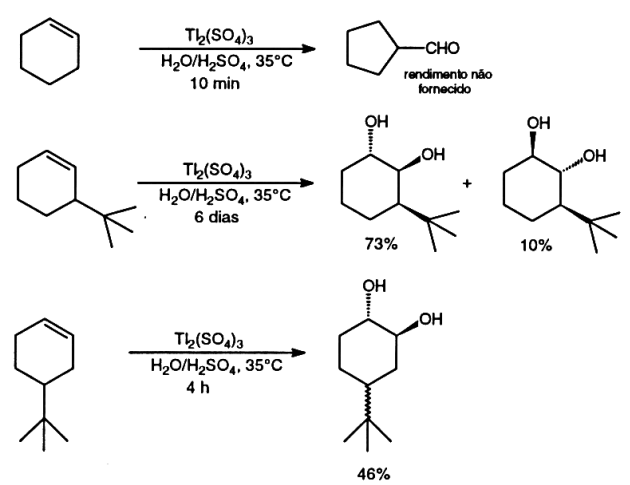

Esquema 14

para a obtenção de produtos de adição diferentes de 1,2-dióis ${ }^{53,54}$. Barluenga et al. ${ }^{53}$, por exemplo, realizaram a adição de aminas aromáticas ao cicloexeno, na presença de TTA (Equação 12).

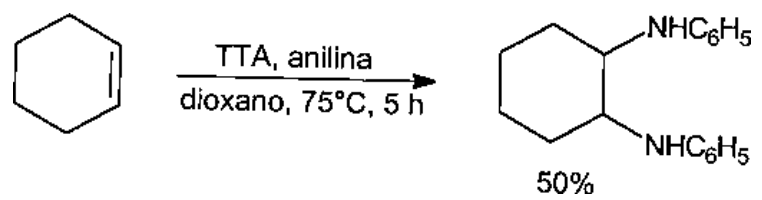

A contração do cicloexeno com tálio(III) em solução de $\mathrm{HClO}_{4}$ foi realizada com bom rendimento por Halpern et al. ${ }^{55}$, em 1973. Contudo, a reação do 1-metilcicloexeno levou a uma mistura do produto de contração e do 1,2-diol (Esquema 15).

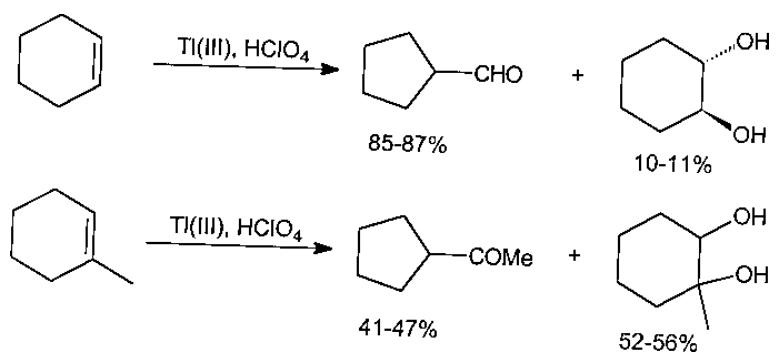

Esquema 15

Os autores propuseram, nesse artigo, um mecanismo que passaria por um carbocátion livre (Esquema 16).

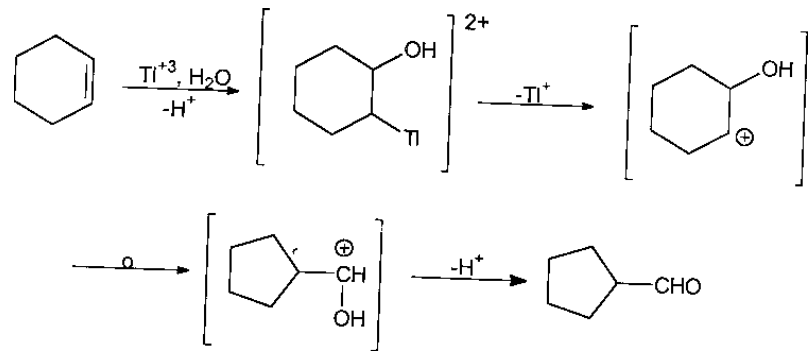

Esquema 16

McKillop et al. ${ }^{56,57}$ realizaram a contração do cicloexeno com TTN, utilizando metanol como solvente. Os resultados foram superiores aos obtidos anteriormente. Cicloexenos substituídos também levaram aos respectivos produtos de contração, com rendimentos de razoáveis a bons (Esquema 17).

Neste mesmo trabalho, a reação do cicloexeno e do 3metilcicloexeno com TTFA em éter também foi relatada. Os produtos de contração foram obtidos em 66 e 57\%, respectivamente.

$\mathrm{O}$ mesmo grupo de pesquisa mostrou que a contração do cicloexeno pode ser realizada com $85 \%$ de rendimento, utilizando TTN/K-10 como oxidante ${ }^{35}$ (Equação 13). 


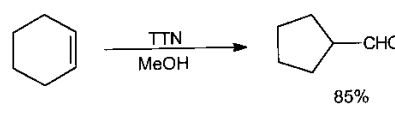

$\longrightarrow \frac{\mathrm{TTN}}{\mathrm{MeOH}} \rightarrow \sum_{64 \%}^{\mathrm{CHO}}$
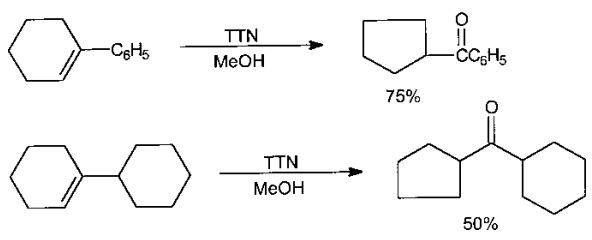

Esquema 17

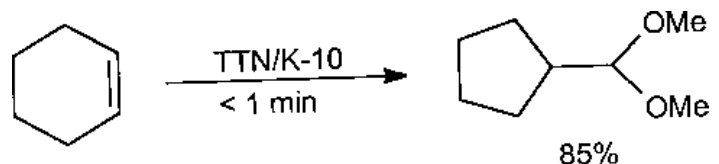

\section{Biciclos e Policiclos}

Corey et al ${ }^{58,59}$ utilizaram o TTN na preparação de um intermediário chave para a síntese de 11-desoxiprostaglandinas. Segundo os autores, as tentativas de aplicação do método de McKillop et al. ${ }^{56}$ (cf. Esquema 17) sempre produziram uma complexa mistura de produtos. A melhor condição encontrada foi quando se utilizou solução aquosa de ácido perclórico (Equação 14).<smiles>O=C1C[C@H]2C=CCC[C@H]2O1</smiles>

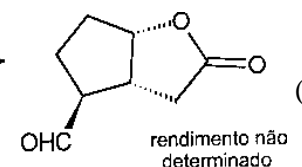

A contração do biciclo 12 com TTN levou a uma mistura diastereomérica dos aldeídos 13, em $66 \%$ de rendimento ${ }^{60}$ (Equação 15).<smiles>O=C(OCc1ccccc1)N1CC[C@H]2CC=CC[C@H]2C1</smiles>

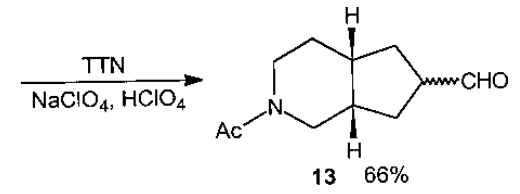

Em um trabalho publicado em 1978 , Sekizaki et al. ${ }^{61}$ mostraram que o aumento da temperatura desfavorece a formação do produto de contração do tujopseno (Esquema 18), contrastando com os resultados anteriormente obtidos por Lee e Price $^{50}$ (cf. Esquema 13).

A reação de olefinas esteroidais não foi tão estudada quanto a das correspondentes cetonas, e, além disso, não se observaram produtos de contração em nenhum dos diversos exemplos estudados ${ }^{62-64}$.

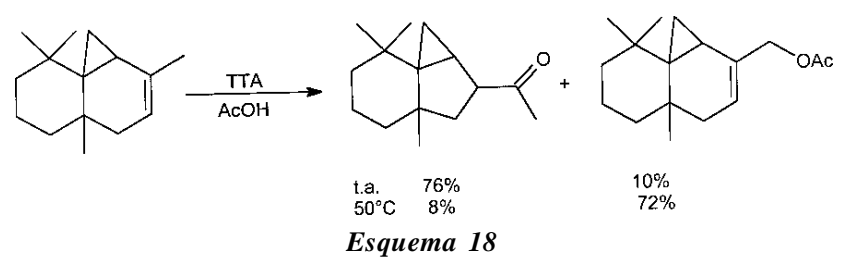

\section{Heterociclos}

A reação do 3,4-diidro-2H-pirano com tálio(III) foi estudada em condições similares por três grupos de pesquisa. Todos eles utilizaram como oxidante TTN e, como solvente, metanol. McKillop et al. ${ }^{57}$ obtiveram o 2-(dimetoximetil)tetraidrofurano em $65 \%$ de rendimento, enquanto que Antus et al. ${ }^{65}$ conseguiram um rendimento de $77 \%$ do produto. Posteriormente, Kaye et al. ${ }^{66}$ prepararam o mesmo composto em rendimento quantitativo (Esquema 19).

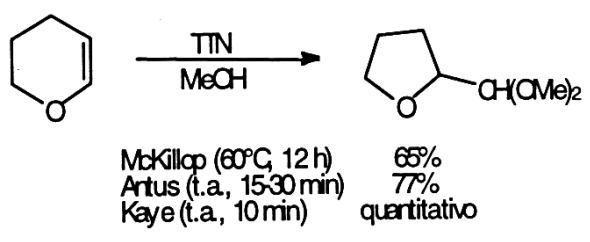

Esquema 19

A reação do 3,4-diidro- $2 H$-pirano com TTA em tiocianato de potássio e metanol foi estudada por Mitani et al. ${ }^{67}$. Neste caso, um tiocianato foi obtido com baixo rendimento $(8 \%)$.

A oxidação de 2,2-dimetil- $2 H$-cromenos com TTN levou a produtos de contração em rendimentos de moderados a bai$\operatorname{xos}^{65}$ (Esquema 20).
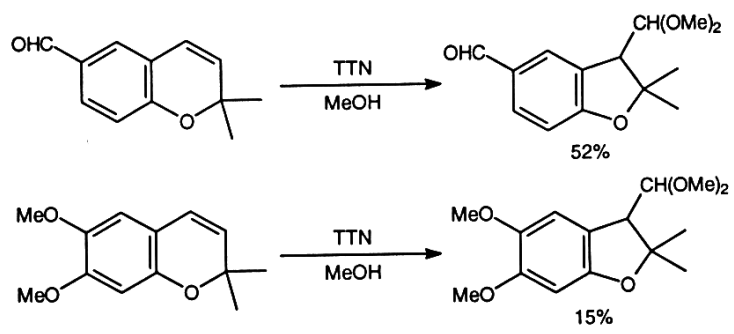

Esquema 20

Por outro lado, ocorreu exclusivamente a formação de dióis cis quando se reagiram outros cromenos com $\operatorname{TTN}^{68}$ (Esquema 21).

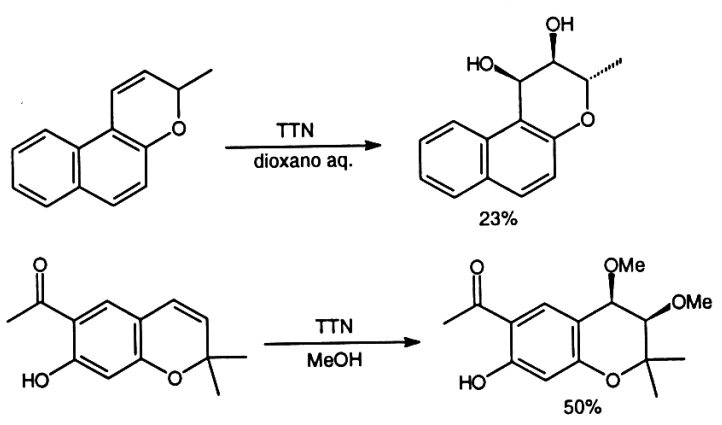

Esquema 21

A síntese esterosseletiva de tetraidrofuranos foi realizada através de uma reação de contração de anel, utilizando como oxidante TTN $^{66,69}$ (Esquema 22). Foram empregados dois solventes diferentes: acetonitrila ${ }^{66}$ e metanol ${ }^{69}$.

\section{Dienos}

Da mesma forma que olefinas, dienos também reagem com sais de tálio(III) levando a produtos de contração e/ou adição.

Murakami e Nishida ${ }^{70}$ realizaram a reação do cicloexadieno com TTN em uma mistura de metanol e diclorometano, obtendo o produto de contração em $31 \%$, juntamente com dois produtos de adição (Equação 16). Outros pesquisadores também estudaram a reação do mesmo substrato com TTA e TTFA, chegando somente aos produtos de $\operatorname{adição~}^{71-73}$. 


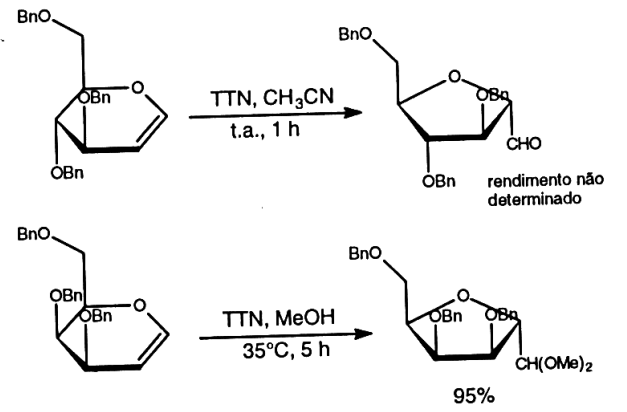

Esquema 22

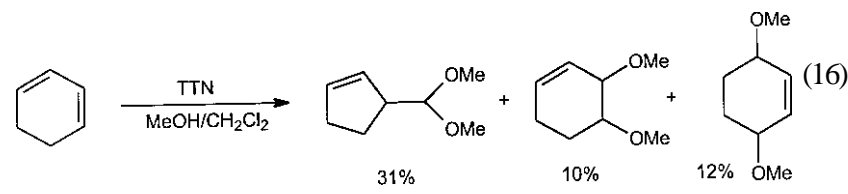

Posteriormente, Rigby e Pigge publicaram dois artigos sobre a reação de dienos com TTN em metanol ${ }^{74,75}$.

O dieno 14 levou a um único produto, em $85 \%$ de rendimento ${ }^{74}$, permitindo a síntese total da $(+)$-ferruginina (Esquema 23). Neste trabalho, também foi realizada a contração de outros dienos.

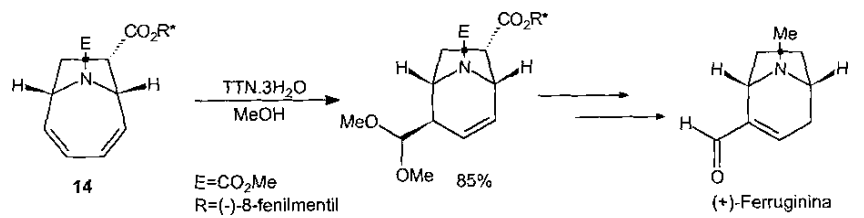

Esquema 23

Por outro lado, a reação dos dienos 15a e b, nas mesmas condições, forneceu o produto de contração, juntamente com o 1,4-diol metoxilado ${ }^{75}$ (Esquema 24). Os motivos que causam a diferença de comportamento entre os dienos 14 e 15 frente à reação com TTN não estão esclarecidos.

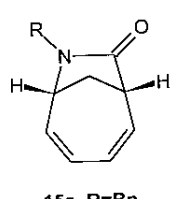

$15 \mathrm{a}, \mathrm{R}=\mathrm{Bn}$

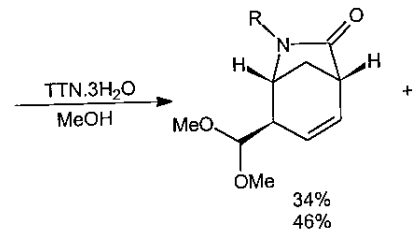

Esquema 24
A reação de outros dienos cíclicos com TTA e TTN também foi estudada, porém não foram obtidos produtos de contração ${ }^{76,77}$.

\section{Outros Cicloalcenos}

Ao contrário do observado com as cetonas, cuja contração foi realizada quase que exclusivamente em anéis de seis membros, a contração de diversos cicloalcenos já foi estudada.

McKillop et al ${ }^{56,57}$ realizaram a contração do cicloepteno e do cicloocteno em duas condições diferentes. Ao ser tratado com TTN em metanol, o cicloepteno originou o cicloexanocarboxaldeído em $86 \%$ de rendimento; já o cicloeptanocarboxaldeído foi formado em apenas $21 \%$, a partir do cicloocteno. Utilizando TTFA em éter, os mesmos produtos foram obtidos em rendimentos mais baixos (Esquema 25).

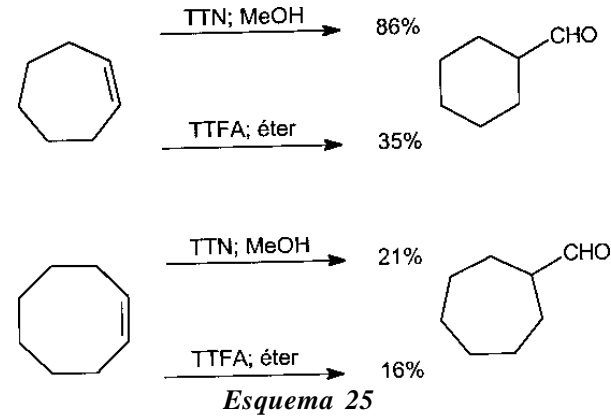

Quase que simultaneamente, outro grupo de pesquisa publicou seus estudos sobre a contração de diversos cicloalcenos ${ }^{55,78}$. As reações foram realizadas em solução de ácido perclórico, propiciando a contração de ciclobutenos, -eptenos e -octenos. Somente com os ciclopentenos o rearranjo oxidativo que leva à contração não foi o caminho predominante (Tabela 4).

Um interessante resultado mostrado na tabela 4 é que, em contraste com o comportamento do ciclopenteno, o 1-metilciclopenteno leva ao produto de contração, a ciclobutilmetilcetona. Neste caso, a estabilização adicional, devida ao substituinte metila, do carbocátion formado na contração é, aparentemente, suficiente para compensar (ao menos em uma pequena proporção) a energia torsional associada com a formação do anel de 4 membros.

Sasaki et al. ${ }^{79}$ realizaram a reação do triciclo $\mathbf{1 6}$ com TTA e TTN. Ao contrário do normalmente observado em reações de contração de anel promovidas por tálio(III), o melhor resultado foi obtido quando se utilizou TTA em ácido acético (Equação 17).
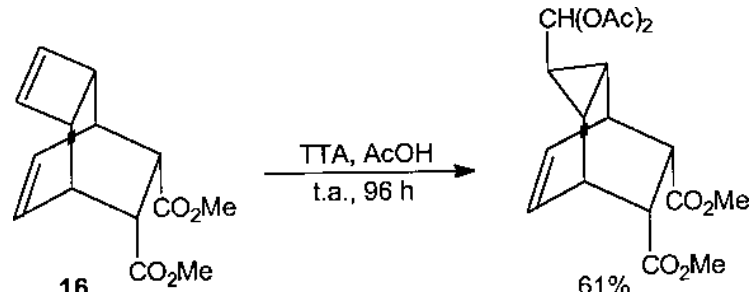

Utilizando a metodologia desenvolvida por McKillop et al. ${ }^{57}$ (cf. Esquema 17), Rigby e Kirova-Snover ${ }^{80}$ realizaram, em 1997, a síntese do $\beta$-cedreno, através da contração de um cicloepteno (Esquema 26). Cabe ressaltar que os autores

Tabela 4. Reação de Cicloalcenos com Tálio(III) em Solução Aquosa de $\mathrm{HClO}_{4}$.

\begin{tabular}{llcc}
\hline Alceno & Produto carbonílico & \multicolumn{2}{c}{ Rendimentos } \\
& & Carbonílico & 1,2 -diol \\
\hline ciclobuteno & ciclopropanocarboxaldeído & $75-85$ & - \\
ciclopenteno & ciclopentanona & $21-27$ & $72-75$ \\
cicloepteno & cicloexanocarboxaldeído & $81-83$ & $9-11$ \\
1-metilciclobuteno & ciclopropilmetilcetona & $88-92$ & - \\
1-metilciclopenteno & ciclobutilmetilcetona & $16-24$ & $76-79$ \\
1-metilcicloepteno & cicloexilmetilcetona & $86-92$ & $5-10$ \\
\hline
\end{tabular}


tentaram, sem sucesso, a contração de um dieno, de modo análogo ao realizado na síntese da (+)-ferruginina ${ }^{74}$ (cf. Esquema 23).

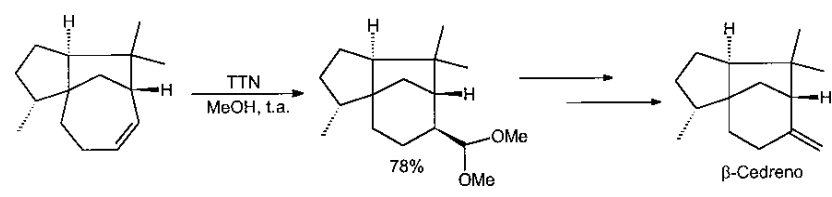

Esquema 26

Recentemente, a reação de contração de uma série de álcoois homoalílicos, utilizando TTN em ácido acético/água, foi estudada por nós ${ }^{81}$.

\section{CONCLUSÃO}

Conforme mostrado neste trabalho, o uso de sais de tálio(III) continua sendo um método viável, juntamente com as conhecidas reações de Favorskii e de Wolf, para promover a contração de anel de diversos tipos de compostos cíclicos. Dentre estas contrações, destaca-se a construção de ciclopentanos funcionalizados, que estão presentes em muitos produtos naturais.

\section{AGRADECIMENTOS}

Os autores agradecem à FAPESP, à CAPES e ao CNPq pelos auxílios e bolsas concedidos.

\section{REFERÊNCIAS}

1. Ferraz, H. M. C.; Silva Jr., L. F.; Vieira, T. de O.; Synthesis 1999, 2001.

2. Taylor, E. C.; McKillop, A.; Acc. Chem. Res. 1970, 3, 338.

3. Rawlinson, D. J.; Sosnovsky, G. Synthesis 1973, 567.

4. McKillop, A.; Taylor, E. C.; Adv. Organomet. Chem. 1973, 11, 147.

5. McKillop, A.; Taylor, E. C.; Chem. Ber. 1973, 9, 4.

6. McKillop, A.; Pure Appl. Chem. 1975, 463.

7. McKillop, A.; Taylor, E. C.; Endeavor 1976, 35, 88.

8. McKillop, A.; Taylor, E. C. In Comprehensive Organomet. Chem.; Wilkinson, G., Ed.; Pergamon Press: New York, 1982; Vol. 7, p 465.

9. Uemura, S. In Synthetic Reagents; Horwood, E., Ed.; J. J. Pizey: 1983; Vol. 5, p 164.

10. Butler, R. N.; Chem. Rev. 1984, 84, 249.

11. McKillop, A.; Taylor, E. C. In Organic Synthesis by Oxidation with Metal Compounds; Miss, W. J., De Jonge, Cornelis, R. H. I., Eds.; Plenum: New York, 1986, p 695.

12. Ferraz, H. M. C.; Quim. Nova 1987, 10, 106.

13. Merkushev, E. B.; Synthesis 1988, 923.

14. Usyatinskii, A. Y.; Bregadze, V. I.; Russ. Chem. Rev. 1988, 57, 1054

15. Ferraz, H. M. C.; Quim. Nova 1989, 12, 155.

16. Ferraz, H. M. C.; Ribeiro, C. M. R.; Quim. Nova 1990, 13,88 .

17. Douglas, K. T.; Bunni, M. A.; Baindur, S. R.; Int. J. Biochem. 1990, 22, 429.

18. Markó, I. E.; Leung, C. W. In Comprehensive Organomet. Chem.; Abel, E. W., Stone, F. G. A., Wilkinson, G., Eds.; Pergamon Press: New York, 1994; Vol. 11, p 437.

19. Prakash, O.; Aldrichimica Acta 1995, 28, 63.

20. Wiberg, K. B.; Koch, W.; Tetrahedron Lett. 1966, 1779.

21. McKillop, A.; Hunt, J. D.; Taylor, E. C.; J. Org. Chem. 1972, 37, 3381.

22. Kabbe, H. -J.; Ann. 1962, 656, 204.

23. Littler, J. S.; J. Chem. Soc. 1962, 827.

24. Khanna, M. S.; Garg, C. P.; Kapoor, R. P.; Synlett 1992, 393.
25. Lee, J. C.; Park, C.; Choi, Y.; Synth. Commun. 1997, 27, 4079 .

26. Kuehne, M. E.; Giacobbe, T. J.; J. Org. Chem. 1968, $33,3359$.

27. Corbani, F.; Rindone, B.; Scolastico, C.; Tetrahedron 1973, 29, 3253.

28. Antus, S.; Boross, F.; Kajtár-Peredy, M.; Radics, L.; Nógradi, M.; Liebigs Ann. Chem. 1980, 1283.

29. Ferraz, H. M. C.; Silva Jr, L. F.; Tetrahedron Lett. 1997, $38,1899$.

30. Bird, C. W.; Cooper, R.; Org. Prep. Proced. Int. 1993, 25,237

31. Cabe ressaltar que não tivemos acesso ao artigo, mas apenas ao seu abstract.

32. Ferraz, H. M. C.; Silva Jr, L. F.; J. Org. Chem. 1998, 63,1716

33. Mincione, E.; Bovicelli, P.; Gil, J. B.; Forcellese, M. L.; Gazz. Chim. Ital. 1985, 115, 37.

34. Banerjee, A. K.; Carrasco, M. C.; Peña-Matheud, C. A.; Recl. Trav. Chim. Pays-Bas 1989, 108, 94.

35. Taylor, E. C.; Chiang, C. -S.; McKillop, A.; White, J. F.; J. Am. Chem. Soc. 1976, 98, 6750.

36. Miles, D. H.; Lho, D. -S.; Chittawong, V.; Payne, A. M.; J. Org. Chem. 1990, 55, 4034.

37. Ciattini, G.; Morera, E.; Ortar, G.; J. Heterocyclic Chem. 1982, 19, 395.

38. Khanna, M. S.; Singh, O. V.; Garg, C. P.; Kapoor, R. P.; Synth. Commun. 1993, 23, 585.

39. Grisar, J. M.; Bolkenius, F. N.; Petty, M. A.; Verne, J.; J. Med. Chem. 1995, 38, 453.

40. Irwin, A. J.; Jones, J. B.; J. Org. Chem. 1977, 42, 2176.

41. Just, G.; Donnini, G. P.; Can. J. Chem. 1977, 55, 2998.

42. Romeo, A.; Ortar, G.; Tetrahedron Lett. 1972, $28,5337$.

43. Ortar, G.; Romeo, A.; J. Chem. Soc., Perkin Trans. 1 1976, 111.

44. Mincione, E.; Barraco, P.; Forcellese, M. L.; Gazz. Chim. Ital. 1980, 110, 515.

45. Maione, A. M.; Romeo, A.; Cerrini, S.; Fedeli, W.; Mazza, F.; Tetrahedron 1981, 37, 1407.

46. Auclair, J. -P.; Gramain, J. -C.; J. Chem. Soc., Perkin Trans. 1 1988, 23.

47. Salaun, J.; Garnier, B.; Conia, J. M.; Tetrahedron 1974, 30,1423

48. Anderson, C. B.; Winstein, S.; J. Org. Chem. 1963, 28, 605.

49. Lee, J. B.; Price, M. J.; Tetrahedron Lett. 1962, 1155.

50. Lee, J. B.; Price, M. J.; Tetrahedron 1964, 20, 1017.

51. Freppel, C.; Favier, R.; Richer, J. -C.; Zador, M.; Can. J. Chem. 1971, 49, 2586.

52. Freppel, C.; Favier, R.; Richer, J. -C.; Zador, M.; Can. J. Chem. 1971, 49, 2590.

53. Aranda, V. G.; Barluenga, J.; Aznar, F.; Synthesis 1974, 504.

54. Emmer, G.; Zbiral, E.; Liebigs Ann. Chem. 1979, 796.

55. Abley, P.; Byrd, J. E.; Halpern, J.; J. Am. Chem. Soc. 1973, 95, 2591.

56. McKillop, A.; Hunt, J. D.; Taylor, E. C.; Kienzle, F.; Tetrahedron Lett. 1970, 5275.

57. McKillop, A.; Hunt, J. D.; Kienzle, F.; Bigham, E.; Taylor, E. C.; J. Am. Chem. Soc. 1973, 95, 3635.

58. Corey, E. J.; Ravindranathan, T.; Tetrahedron Lett. 1971 , 4753.

59. Corey, E. J.; Snider, B. B.; J. Org. Chem. 1974, 39, 256.

60. Holick, W.; Jenny, E. F.; Heusler, K.; Tetrahedron Lett. 1973, 3421 .

61. Sekizaki, H.; Ito, M.; Inoue, S.; Bull. Chem. Soc. Jpn. 1978, 51, 2439.

62. Cocton, B.; de Paulet, A. C.; Bull. Soc. Chim. Fr. 1966, 2947.

63. Glotter, E.; Schwarts, A.; J. Chem. Soc., Perkin Trans. 1 1976, 1660. 
64. Schwartz, A.; Glotter, E.; J. Chem. Soc., Perkin Trans. 1 1977, 2470.

65. Antus, S.; Gottsegen, A.; Nógrádi, M.; Gergely, A.; Chem. Ber. 1979, 112, 3879 .

66. Kaye, A.; Neidle, S.; Reese, C. B.; Tetrahedron Lett. 1988, 29, 1841.

67. Mitani, H.; Ando, T.; Yukawa, Y.; Chem. Lett. 1972, 455.

68. Begley, M. J.; Mohamed, S. E.; Whiting, D. A.; D'Souza, F.; Hatam, N. A. R.; J. Chem. Soc., Perkin Trans. 1 1983, 883.

69. Bettelli, E.; D’Andrea, P.; Mascanzoni, S.; Passacantilli, P.; Piancatelli, G.; Carbohydr. Res. 1998, 306, 221.

70. Murakami, M.; Nishida, S.; Chem. Lett. 1981, 997.

71. Uemura, S.; Tabata, A.; Okano, M.; Ichikawa, K.; J. Chem. Soc., Chem. Commun. 1970, 1630.

72. Emmer, G.; Zbiral, E.; Tetrahedron 1977, 33, 1415.
73. Uemura, S.; Miyoshy, H.; Tabata, A.; Okano, M.; Tetrahedron 1981, 37, 291.

74. Rigby, J. H.; Pigge, F. C.; J. Org. Chem. 1995, 60, 7392.

75. Rigby, J. H.; Pigge, F. C.; Synlett 1996, 631.

76. Bach, R. D.; Holubka, J. W.; J. Am. Chem. Soc. 1974, 96, 7814.

77. Bach, R. D.; Holubka, J. W.; Willis, C. L.; J. Am. Chem. Soc. 1982, 104, 3980.

78. Byrd, J. E.; Cassar, L.; Eaton, P. E.; Halpern, J.; J. Chem. Soc., Chem. Commun. 1971, 40.

79. Sasaki, T.; Kanematsu, K.; Kondo, A.; Okada, K.; J. Org. Chem. 1976, 41, 2231.

80. Rigby, J. H.; Kirova-Snover, M.; Tetrahedron Lett. 1997, $38,8153$.

81. Ferraz, H. M. C.; Santos, A. P.; Silva Jr., L. F.; Vieira, T. de O.; Synth. Commun., no prelo. 